to appear in J. Phys. A

15 November 2000

quant-ph/0105087

\title{
FROM GAUGE TRANSFORMATIONS TO TOPOLOGY COMPUTATION IN QUANTUM LATTICE GAS AUTOMATA
}

\author{
David A. Meyer \\ Project in Geometry and Physics \\ Department of Mathematics \\ University of California/San Diego \\ La Jolla, CA 92093-0112 \\ dmeyer@chonji.ucsd.edu \\ and \\ Institute for Physical Sciences \\ Los Alamos, NM 87544
}

\begin{abstract}
The evolution of a quantum lattice gas automaton (LGA) for a single charged particle is invariant under multiplication of the wave function by a global phase. Requiring invariance under the corresponding local gauge transformations determines the rule for minimal coupling to an arbitrary external electromagnetic field. We develop the Aharonov-Bohm effect in the resulting model into a constant time algorithm to distinguish a one dimensional periodic lattice from one with boundaries; any classical deterministic LGA algorithm distinguishing these two spatial topologies would have expected running time on the order of the cardinality of the lattice.
\end{abstract}

PACS numbers: 03.65.Lx, 03.65.Vf, 03.65.Pm.

KEY WORDS: quantum lattice gas; quantum computation; Aharonov-Bohm effect; spatial topology. 
Quantum lattice gas automata (QLGA) have been proposed as a possible architecture for solid state quantum computers since they require only an array of sites which can support an extended (multi-)electron wave function [1]. The simplicity of such an architecture makes nanoscale fabrication plausible [2]. The main incentives for pursuing the program of quantum computation, however, are the quantum algorithms of Shor [3] and Grover [4], for example, which provide substantial improvements over deterministic or probabilistic algorithms. These quantum algorithms can be efficiently implemented with quantum gate arrays [5], but at least in simple translations - analogous to deterministic billiard ball models for universal computation [6] - seem impractical to implement in QLGA models. Just as deterministic LGA efficiently simulate fluid flow in certain parameter regimes [7], QLGA seem best suited for simulation of quantum physical processes $[1,8]$. Single particle QLGA have been shown, in fact, to limit to the Schrödinger [9] and Dirac [1] equations under appropriate conditions. In this letter we show that in simulating a single quantum particle, QLGA efficiently perform a new and interesting computation - of spatial topology.

We draw inspiration from the topological invariance of the spectral flow in a one parameter family of Dirac operators [10]. Spectral flow derives from the components of the group of gauge transformations, so after recalling the definition of one dimensional single particle QLGA with inhomogeneous potentials, we analyze which evolution rules are gauge equivalent. We find a difference between the gauge equivalence classes of rules on periodic lattices and lattices with boundary, and show how this may be exploited to distinguish between these two spatial topologies. We conclude with a discussion of the complexity of this quantum computation and point out directions for further investigation.

A one particle QLGA is a discrete time model for a quantum particle moving in an array (lattice) of sites. Here we will consider only finite one dimensional lattices $L$, with or without periodic boundary conditions. In these cases we need only allow two velocities $\{ \pm 1\}$ in order to construct a model which limits to the Schrödinger [9] or Dirac [1] equation as the timestep and lattice spacing scale to zero appropriately. The amplitudes for the particle to be (left,right) moving at a lattice site $x \in L$ combine into a two component complex vector $\psi(t, x):=\left(\psi_{-1}(t, x), \psi_{+1}(t, x)\right)$ which evolves as

$$
\psi(t+1, x)=w_{-1}(x) \psi(t, x-1)+w_{+1}(x) \psi(t, x+1) .
$$

Here the weights $w_{\beta}(x) \in M_{2}(\mathbb{C})$ are $2 \times 2$ complex matrices constrained by the requirement that the global evolution matrix

$$
U:=\left(\begin{array}{ccccccc}
\ddots & & & & & & \\
& w_{-1}(x-1) & 0 & w_{+1}(x-1) & & \\
& & w_{-1}(x) & 0 & w_{+1}(x) & & \\
& & & w_{-1}(x+1) & 0 & w_{+1}(x+1) & \\
& & & & & & \ddots
\end{array}\right)
$$

be unitary. 
For periodic boundary conditions the top and bottom rows (of blocks) of $U$ wrap around and completely homogeneous solutions are possible. We showed in [1] that the most general parity invariant homogeneous solutions, up to unitary equivalence and an overall unobservable phase, form a one parameter family

$$
w_{-1}(x)=w_{-1}:=\left(\begin{array}{cc}
0 & i \sin \theta \\
0 & \cos \theta
\end{array}\right) \quad w_{+1}(x)=w_{+1}:=\left(\begin{array}{cc}
\cos \theta & 0 \\
i \sin \theta & 0
\end{array}\right)
$$

where $\theta$ scales to the mass in the Dirac equation limit.

Without periodic boundary conditions, the local rule must change at the boundary. We show in [11] that there is a one parameter family of (Type II) boundary conditions which give the global evolution matrix the form:

$$
U:=\left(\begin{array}{cccc}
0 & \bar{w}_{+1} & & \\
w_{-1} & 0 & w_{+1} & \\
& w_{-1} & 0 & \\
& & & \ddots
\end{array}\right)
$$

at the left boundary. Here

$$
\bar{w}_{+1}:=\left(\begin{array}{cc}
0 & 0 \\
i e^{i \zeta} & 0
\end{array}\right)
$$

for any $\zeta \in \mathbb{R}$ and the right boundary condition is characterized by $\bar{w}_{-1}$ obtained by parity transformation, although with an independent phase. For simplicity we set both phase angles to zero in the following discussion; this will not affect the relevant features of our results.

The global evolution matrix $U$ acts by left multiplication $\Psi(t+1)=U \Psi(t)$ on the wave function $\Psi(t): L \times\{ \pm 1\} \longrightarrow \mathbb{C}$. Multiplication of the wave function by an overall phase $e^{-i \alpha}$ leaves the quantum state invariant since the phase cancels in any observable $\langle\Psi|\mathcal{O}| \Psi\rangle$. Requiring invariance of the evolution under the local gauge transformations $e^{-i \alpha(t, x)}$ corresponding to this global symmetry necessitates modification of $U$ : Setting $\psi^{\prime}(t, x):=e^{-i \alpha(t, x)} \psi(t, x)$, which we write as

$$
\Psi^{\prime}(t):=D\left[e^{-i \alpha(t, x)}\right] \Psi(t)
$$

we achieve gauge invariance with

$$
U^{\prime}(t):=D\left[e^{-i \alpha(t+1, x)}\right] U D\left[e^{i \alpha(t, x)}\right]
$$

since

$$
\begin{aligned}
U^{\prime}(t) \Psi^{\prime}(t) & =D\left[e^{-i \alpha(t+1, x)}\right] U D\left[e^{i \alpha(t, x)}\right] D\left[e^{-i \alpha(t, x)}\right] \Psi(t) \\
& =D\left[e^{-i \alpha(t+1, x)}\right] \Psi(t+1) \\
& =\Psi^{\prime}(t+1)
\end{aligned}
$$


Expanding $(3 b)$ we find that the superdiagonal blocks of $U^{\prime}(t)$ are

$$
U^{\prime}(t)_{x-1, x}=e^{-i \alpha(t+1, x-1)+i \alpha(t, x)} w_{+1},
$$

while the subdiagonal blocks are

$$
U^{\prime}(t)_{x, x-1}=e^{-i \alpha(t+1, x)+i \alpha(t, x-1)} w_{-1} .
$$

For a periodic lattice, $x$ runs over all the labels $\{0, \ldots,|L|-1\}$ of the lattice sites and the indices are interpreted mod $|L|$. For a lattice with boundaries, $x \in\{1, \ldots,|L|-1\}$ since there are no blocks in the evolution matrix containing nonzero amplitudes for transitions between $x=0$ and $x=|L|-1$. Multiplying and dividing each block in column $x$ (with these same conventions) of $U^{\prime}(t)$ by $e^{i \alpha(t+1, x)}$, the expressions in (4) can be rewritten as

$$
U^{\prime}(t)_{x-1, x}=e^{-i \Delta_{t} \alpha(t+1, x)+i \Delta_{x} \alpha(t+1, x)} w_{+1}
$$

and

$$
U^{\prime}(t)_{x, x-1}=e^{-i \Delta_{t} \alpha(t+1, x-1)-i \Delta_{x} \alpha(t+1, x)} w_{-1},
$$

where $\Delta$ is the difference operator so $\Delta_{t} \alpha(t+1, x):=\alpha(t+1, x)-\alpha(t, x)$ and similarly for $\Delta_{x}$. In column $x$ both the super- and subdiagonal blocks now contain the phase factor $e^{-i \Delta_{t} \alpha(t+1, x)}$. Recall that when we included an inhomogeneous potential in a QLGA [12] it also had the effect of multiplying the blocks in column $x$ by a phase factor, $e^{-i \phi(x)} .^{*}$ Thus the gauge transformation (3) transforms a potential $\phi(t, x)$ to

$$
\phi^{\prime}(t, x)=\phi(t, x)+\Delta_{t} \alpha(t+1, x) .
$$

The $\Delta_{x} \alpha$ phases in the gauge transformed evolution matrix $U^{\prime}(t)$ remind us that the electromagnetic potential has a second component - the vector potential $A$. The conjugate $\Delta_{x} \alpha$ phases of the antidiagonal blocks in (5) indicate that the vector potential enters the evolution matrix in the same way. That is, in the presence of an electromagnetic potential $(\phi(t, x), A(t, x))$, the super- and subdiagonal blocks in the evolution matrix are

$$
U^{(\phi, A)}(t)_{x-1, x}=e^{-i \phi(t, x)+i A(t, x)} w_{+1}
$$

and

$$
U^{(\phi, A)}(t)_{x, x-1}=e^{-i \phi(t, x)-i A(t, x)} w_{-1}
$$

respectively. Notice that for a lattice with boundaries, $A(t, 0) \equiv A(t,|L|)$ does not enter into these expressions for the global evolution, although it does for a periodic lattice. $U^{(\phi, A)}$ describes the discrete version of the usual minimal coupling of a Dirac particle to an

\footnotetext{
* Column and row are interchangeable here, corresponding to multiplying by the local potential phase factors before or after the advection part of each timestep, respectively. The algorithm described in [12] multiplies before, while those in [9] and [13] do so afterwards. To obtain the expressions corresponding to those in (5), but with a common $\Delta_{t} \alpha$ phase in each row, we can multiply and divide each block in row $x$ of $U^{\prime}(t)$ by $e^{i \alpha(t, x)}$.
} 
external (classical) electromagnetic field [14]. The conjugate phases from the vector potential are the consequence of the velocity dependence of this coupling; the vector potential itself transforms to

$$
A^{\prime}(t, x)=A(t, x)+\Delta_{x} \alpha(t+1, x)
$$

under the gauge transformation (3), analogously to the transformation (6) of the scalar potential.

The consequences of this gauge freedom in the vector potential differ for periodic lattices and those with boundaries: Given any vector potential $A(t, x)$, consider the gauge transformation defined by

$$
\alpha(t+1, x)=-\sum_{y=0}^{x} A(t, y) .
$$

With this definition, $\Delta_{x} \alpha(t+1, x)=-A(t, x)$ for $1 \leq x \leq|L|-1$ so that using (9) in (8) gives a gauge transformed $A^{\prime}(t, x)$ which vanishes except at $x=0$. Since the global evolution on a lattice with boundaries does not depend on $A^{\prime}(t, 0)$, (9) gauge transforms the vector potential away completely - there is exactly one gauge equivalence class of vector potentials. On a periodic lattice, however, the global evolution depends on $A^{\prime}(t, 0)(\bmod$ $2 \pi$ ), so there is a one (periodic) parameter family of gauge equivalence classes. Alternatively, the gauge transformation (9) demonstrates that the only gauge invariant quantity which can be constructed from the vector potential is (a function of)

$$
\delta:=\exp i \sum_{x=0}^{|L|-1} A(t, x),
$$

upon which the evolution depends only for a periodic lattice, in which case (10) is the discrete analogue of a Wilson loop variable/holonomy, i.e., a winding number.

Such a gauge invariant quantity manifests itself in the spectrum of the evolution operator: As the phase $\delta$ varies, so does the spectrum of $U^{(\phi, A)}$ —if the lattice is periodic, but not if it has boundaries. Figure 1 shows an example of this spectral flow [10] for a periodic lattice with $N=16$ and $\theta=\pi / 6$. Here $\phi(t, x)$ has been set to 0 by a gauge transformation, and $\delta$ varies from 0 to $2 \pi$. As $\delta$ increases, the frequencies (energies) of positive frequency right/left moving plane waves, i.e., the eigenvectors of $U^{(\phi, A)}$, increase/decrease, respectively. When the 'mass' $\theta=0$, half

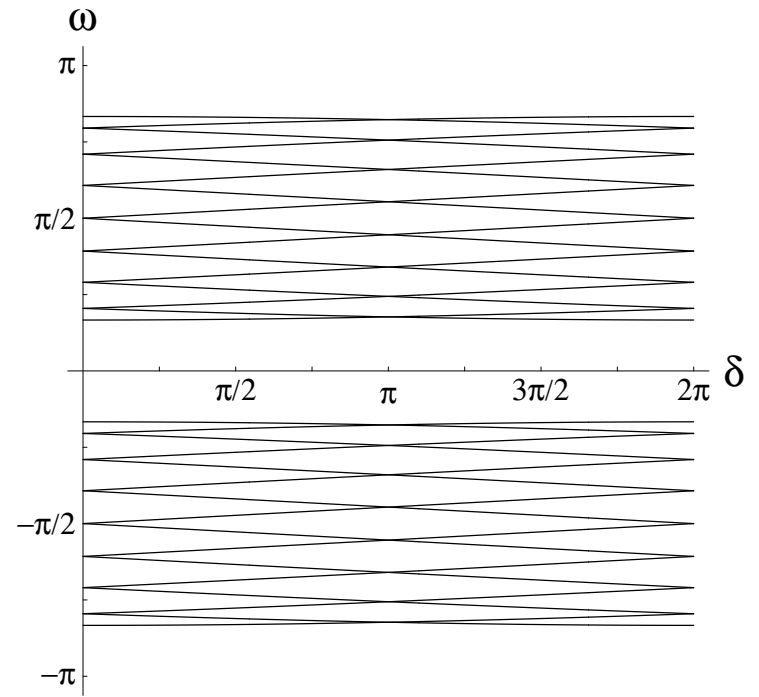

Figure 1. Spectral flow in a periodic QLGA with $|L|=16$ and $\theta=\pi / 6$. The scalar potential has been taken to vanish and $\delta$, the gauge invariant degree of freedom in the vector potential runs from 0 to $2 \pi$. 
the eigenvalues shift up by 1 and half shift down by 1 as $\delta$ varies from 0 to $2 \pi$; the spectral flow (the number of eigenvalues crossing each frequency level) is 2 .

To observe such a spectral flow, imagine preparing a quantum particle on a periodic lattice in a positive frequency, right moving eigenstate of $U^{(0,0)}$, but then applying an external vector potential so that the evolution is by $U^{(0, A)}$ instead, where $A(t, x) \equiv A$. A massless particle will remain in the original eigenstate, but a frequency (energy) measurement at any subsequent timestep will return the original value plus $A=\delta /|L|$. For a massive (i.e., $\theta \neq 0$ ) particle, the initial state is a superposition of the positive and negative frequency eigenstates of $U^{(0, A)}$ with the original wave number, but for small masses the positive frequency still dominates so again a frequency (energy) measurement will return the original value plus an amount on the order of $A$.

Our goal, however, is to distinguish between a periodic lattice and one with boundaries. Since the single particle eigenstates differ for these two situations even if $|L|$ is the same $[13,11]$, without knowing the spatial topology a priori we cannot prepare a quantum particle in an eigenstate. But we can prepare a positive frequency right moving wave packet with bounded support - identically on either lattice. Subsequent frequency measurements will return a distribution of frequencies concentrated around the expectation value. This distribution is the same for either lattice, if the evolution is by $U^{(0,0)}$. But if the evolution is by $U^{(0, A)}$ with $A>0$, say, the observed frequency distribution will be shifted to larger values on the periodic lattice.

Since some finite, fixed, number of measurements suffices to distinguish these distributions with probability $1-\epsilon$ for a given initial wave packet and external vector potential, the computation takes only constant time, independent of the size of the lattice $|L|$. In fact, for larger lattices the initial wave packet can be broader, i.e., more concentrated around its expected energy, and hence fewer measurements are needed to distinguish the original distribution from the one shifted by the vector potential in case the lattice is periodic. In contrast, suppose we try to distinguish a periodic lattice from one with boundaries using a deterministic LGA. Since no superpositions of states are possible, all that we can do is to start a single particle off in one direction and see if it ever changes velocity by reflecting from a boundary. To do so the particle must travel for $O(|L|)$ timesteps, on the average, so the QLGA algorithm provides a even greater improvement over the classical algorithm than does Grover's algorithm for searching [4].

In conclusion, we remark that our algorithm exploits the Aharonov-Bohm effect [15] which is more usually discussed in two dimensions - we are imagining creating the vector potential by applying a magnetic field which threads a (possibly incomplete, in the nonperiodic case) ring of lattice sites. It would be interesting, and potentially useful for pattern recognition [16], to formulate this algorithm for a two dimensional QLGA. Preparation of a localized wave packet, and measurement of its energy, each in constant time, is then plausible on physical grounds, provided that the lattice lies within some fixed area. For the more realistic situation of a fixed density of lattice sites (and hence an increasing spatial area with increasing lattice size) additional analysis is required. As the example of unitary 
transformations and measurements on the Rydberg states of an atom illustrates, careful attention to the details of the physical implementation of a quantum algorithm is required to correctly quantify its computational complexity [17]. Although the evolution of the QLGA can be simulated in the standard poly-local model for quantum computing [18], issues of the time required for state preparation and measurement, and the adiabaticity required when turning on the magnetic field, all must be considered before we can claim to understand how this computation scales physically.

\section{Acknowledgements}

I thank A. P. Balachandran for encouraging me to think about possible QLGA algorithms, Peter Doyle, Mike Freedman and Brosl Hasslacher for useful discussions, and Sun Microsystems for providing computational support. This work was supported in part by the National Security Agency (NSA) and Advanced Research and Development Activity (ARDA) under Army Research Office (ARO) contract number DAAG55-98-1-0376.

\section{References}

[1] D. A. Meyer, "From quantum cellular automata to quantum lattice gases", J. Stat. Phys. 85 (1996) 551-574.

[2] B. Meurer, D. Heitmann and K. Ploog, "Single-electron charging of quantum-dot atoms", Phys. Rev. Lett. 68 (1992) 1371-1374;

G. Springholz, M. Pinczolits, P. Mayer, V. Holy, G. Bauer, H. H. King and L. Salamanca-Riba, "Tuning of vertical and lateral correlations in self-organized PbSe/ $\mathrm{Pb}_{1-x} \mathrm{Eu}_{x}$ Te quantum dot superlattices", Phys. Rev. Lett. 84 (2000) 4669-4672; and references therein.

[3] P. W. Shor, "Algorithms for quantum computation: discrete logarithms and factoring", in S. Goldwasser, ed., Proceedings of the 35th Symposium on Foundations of Computer Science, Santa Fe, NM, 20-22 November 1994 (Los Alamitos, CA: IEEE Computer Society Press 1994) 124-134.

[4] L. K. Grover, "A fast quantum mechanical algorithm for database search", in Proceedings of the 28th Annual ACM Symposium on the Theory of Computing, Philadelphia, PA, 22-24 May 1996 (New York: ACM 1996) 212-219.

[5] See, e.g., D. Beckman, A. N. Chari, S. Devabhaktuni, J. Preskill, "Efficient networks for quantum factoring", Phys. Rev. A 54 (1996) 1034-1063.

[6] N. Margolus, "Physics-like models of computation", Physica D 10 (1984) 81-95; M. Biafore, "Cellular automata for nanometer-scale computation", Physica D $\mathbf{7 0}$ (1994) 415-433.

[7] B. Hasslacher, "Discrete fluids", Los Alamos Science 15 (1988) 175-200, 211-217.

[8] B. M. Boghosian and W. Taylor, IV, "Simulating quantum mechanics on a quantum computer", Physica D 120 (1998) 30-42.

[9] B. M. Boghosian and W. Taylor, IV, "A quantum lattice-gas model for the manyparticle Schrödinger equation in $d$ dimensions", Phys. Rev. E 8 (1997) 705-716. 
[10] M. F. Atiyah, V. K. Patodi and I. M. Singer, "Spectral asymmetry and Riemannian geometry. III", Math. Proc. Camb. Phil. Soc. 79 (1976) 71-99.

[11] D. A. Meyer, "Quantum mechanics of lattice gas automata: Boundary conditions and other inhomogeneities", J. Phys. A 31 (1998) 2321-2340.

[12] D. A. Meyer, "Quantum lattice gases and their invariants", Int. J. Mod. Phys. C 8 (1997) $717-735$.

[13] D. A. Meyer, "Quantum mechanics of lattice gas automata: One-particle plane waves and potentials", Phys. Rev. E 55 (1997) 5261-5269.

[14] P. A. M. Dirac, "The quantum theory of the electron", Proc. Roy. Soc. Lond. A 117 (1928) 610-624.

[15] Y. Aharonov and D. Bohm, "Significance of electromagnetic potentials in the quantum theory", Phys. Rev. 115 (1959) 485-491.

[16] T. Y. Kong and A. Rosenfeld, eds., Topological Algorithms for Digital Image Processing (New York: Elsevier 1996); and references therein.

[17] D. A. Meyer, P. G. Kwiat, R. J. Hughes, P. H. Bucksbaum, J. Ahn and T. C. Weinacht, "Does Rydberg state manipulation equal quantum computation?", Science 289 (1 sep 2000) 1431a.

[18] M. H. Freedman, "Poly-locality in quantum computing", quant-ph/0001077. 